\title{
Anxiety and depression in adolescents with asthma and in their parents. Is an increased basal cholinergic tone a possible further reason to explain the negative impact on asthma control?
}

\author{
Gennaro Liccardi ${ }^{1}$, Luigino Calzetta ${ }^{1,2}$, Manlio Milanese ${ }^{3}$, Maria Beatrice Bilò ${ }^{4}$, Maria Vittoria Liccardi ${ }^{5}$, \\ Maria Gabriella Matera ${ }^{6}$, Paola Rogliani ${ }^{1,2}$
}

${ }^{1}$ Postgraduate School of Respiratory Medicine, Department of Experimental Medicine, University of Rome "Tor Vergata", Rome; ${ }^{2}$ Department of Experimental Medicine, Unit of Respiratory Medicine, University of Rome "Tor Vergata”, Rome; ${ }^{3}$ Division of Pulmonology, S. Corona Hospital, Pietra Ligure; ${ }^{4}$ Allergy Unit, Department of Internal Medicine, University Hospital Ospedali Riuniti, Department of Clinical and Molecular Sciences, Marche Polytechnic University, Ancona; ${ }^{5}$ Psychologist, Naples; ${ }^{6}$ Department of Experimental Medicine, Second University of Naples, Italy

\section{Dear Editor}

We read with interest the excellent article of Licari et al. [1] reporting that anxiety and depression are common in adolescents suffering from asthma as well as in their parents, mainly in mothers. The consequence of this relationship is that emotional disorders might negatively affect also the control of asthma. They suggested,

Corresponding author: Gennaro Liccardi, Postgraduate School of Respiratory Medicine, Department of Experimental Medicine, University of Rome "Tor Vergata", Rome, Italy.

Tel.+39.081.5780554. E-mail: gennaro.liccardi@tin.it

Key words: adolescents; anxiety; asthma control; bronchial asthma; increased cholinergic tone; depression; hypersensitivity; psychological distress.

Authors' contributions: GL, conceived the idea of a "Letter to The Editor", manuscript first draft; MVL, collected bibliographic and clinical data on the "stress" topic; LC, MM, MBB, MVL, MGM, PR, drafted the manuscript and gave input and agreed to the final submitted version. All the authors have read and approved the final version of the manuscript and agreed to be accountable for all aspects of the work.

Conflict of interest: All authors declare that they have no conflict of interest.

Funding: The study has been carried out without any financial support.

Summary statement: The impaired control of asthma symptoms in adolescent with anxiety/depression could be related to an anxiety/stressinduced increased cholinergic tone.

Received for publication: 20 January 2020

Accepted for publication: 6 March 2020.

${ }^{\circ}$ Copyright: the Author(s), 2020

Licensee PAGEPress, Italy

Monaldi Archives for Chest Disease 2020; 90:1233

doi: 10.4081/monaldi.2020.1233

This article is distributed under the terms of the Creative Commons Attribution Noncommercial License (by-nc 4.0) which permits any noncommercial use, distribution, and reproduction in any medium, provided the original author(s) and source are credited. that in clinical practice, the psychological assessment could be included in the asthma work-up.

However, they did not provide comments on the causal and temporal association between anxiety and asthma outcomes. From a general point of view, it has not been fully demonstrated if in asthmatic subjects poor asthma control influences negatively their psychological status or if psychological stress could aggravate asthma symptoms (through several mechanisms). Otherwise, both hypotheses are possible. A fundamental premise is that asthmatic adolescents may experience a period of physical and psychosocial changes that affect their health and well-being. Overall, adolescents with asthma are at increased risk for asthma morbidity, asthma death and even suicidal behaviour. Increased rates of depression and anxiety, in adolescents and their caregivers, can lead to non-adherence to their medical regimens, poor symptom control and poor treatment outcomes. Asthma during adolescence impairs health-related quality of life, especially if the asthma is uncontrolled. It has been also demonstrated that parental stress and air pollution were synergistically associated with increased childhood asthma, indicating a common biological effect of parental stress and air pollution during both prenatal and postnatal periods. Therefore, we would like to suggest some potential mechanisms to explain the correlation between stress/anxiety and asthma in adolescents other than those related to the already mentioned non-adherence to medical regimens or some stress-related excesses (e.g. food, smoke of cigarette / narcotic, etc).

We have previously shown that about $63 \%$ of asthmatic patients reported the usual appearance of at least one non-respiratory symptom (n-RS) before an asthma attack [2]. Anxiety and, to a lesser extent depression, represented the most common n-RSs in our study, suggesting that both anxiety and depression may have a possible role in the development and trigger of an asthma attack. Several studies have shown that psychological stress may enhance bronchial hyperreactivity through different biological mechanisms such as mast cell activation, mediator release, inflammation, impairment of respiratory tolerance. Moreover, it has been also reported a significant correlation between psycho-social stress and stimulation of the cholinergic system, resulting in an increased airway resistance (Figure 1). Visual stimulations (i.e. bloody movies) can rapidly induce (after 1-2 min) vagal-mediated responses associated with airway resistance increase. Indirectly, the results of our study [2] confirm a significant role of the cholinergic 
pathway in the enrolled asthmatic subject. Therefore, we suggest that attention should be focused on the potential role of the parasympathetic system as a trigger of bronchial obstruction at least in asthmatic adolescents reporting the usual onset of cholinergicrelated n-RSs (i.e. stress and/or anxiety) before an asthma attack [3]. In fact, we have hypothesized that, in some individuals, this condition of high basal cholinergic tone might play a predominant role in determining airway obstruction, compared with other wellknown factors such as allergens/air pollutants, infections, exercise, etc. (a definite "asthma phenotype"?).

The vagal hyperactivity induced by anxiety and stress in asthmatics also represents the basis of important considerations by a therapeutic point of view, such as the use of anticholinergic agents. Considering this background, we suggest the need of an adequate phenotyping of stressed asthmatic adolescents who could exhibit an increased basal cholinergic tone. The effect of oxygen and methacholine inhalation, neck suction, slow deep breathing assessed by multiple frequency forced oscillation technique (FOT) as well as measurement of resting heart rate and pupillometry represent the most effective methods for evaluating the level of vagal tone.

According to our previous study [2], a simple question exploring the presence of vagal-related n-RSs during the collection of anamnestic data could help to identify asthmatics with imbalance between sympathetic and parasympathetic systems who could benefit of further diagnostic evaluation of vagal tone. Since the degree of cholinergic tone is likely to be different among asthmatics, we believe it is not possible to rule out that the effectiveness of anticholinergic agents such as tiotropium could be greater in patients with an increased degree of cholinergic tone. This possible increased responsiveness to tiotropium may be usefully exploited also in the event of poor efficacy or occurrence of adverse events with the use of long-acting $\beta_{2}$ agonists (LABAs) [4].

In conclusion, the currently available literature indicates that anxiety and related psychological disorders should be considered as mechanisms that might trigger the airway inflammation, the onset of asthma attacks, and the severity of respiratory symptoms. We concur with Licari et al. [1] and others that adequate educational programs should be planned for those asthmatic patients suffering from psychological disorders (both in adults and minors). We believe that this approach requires a peculiar attention in adolescents in order to obtain a better control of respiratory symptoms in the short term, a delay in asthma progression, and a reduced airway remodelling in the long term.

\section{Acknowledgements}

We thank the biologist Dr. Mariagrazia Iengo and Francesca Lacava for technical assistance in the preparation of this manuscript.

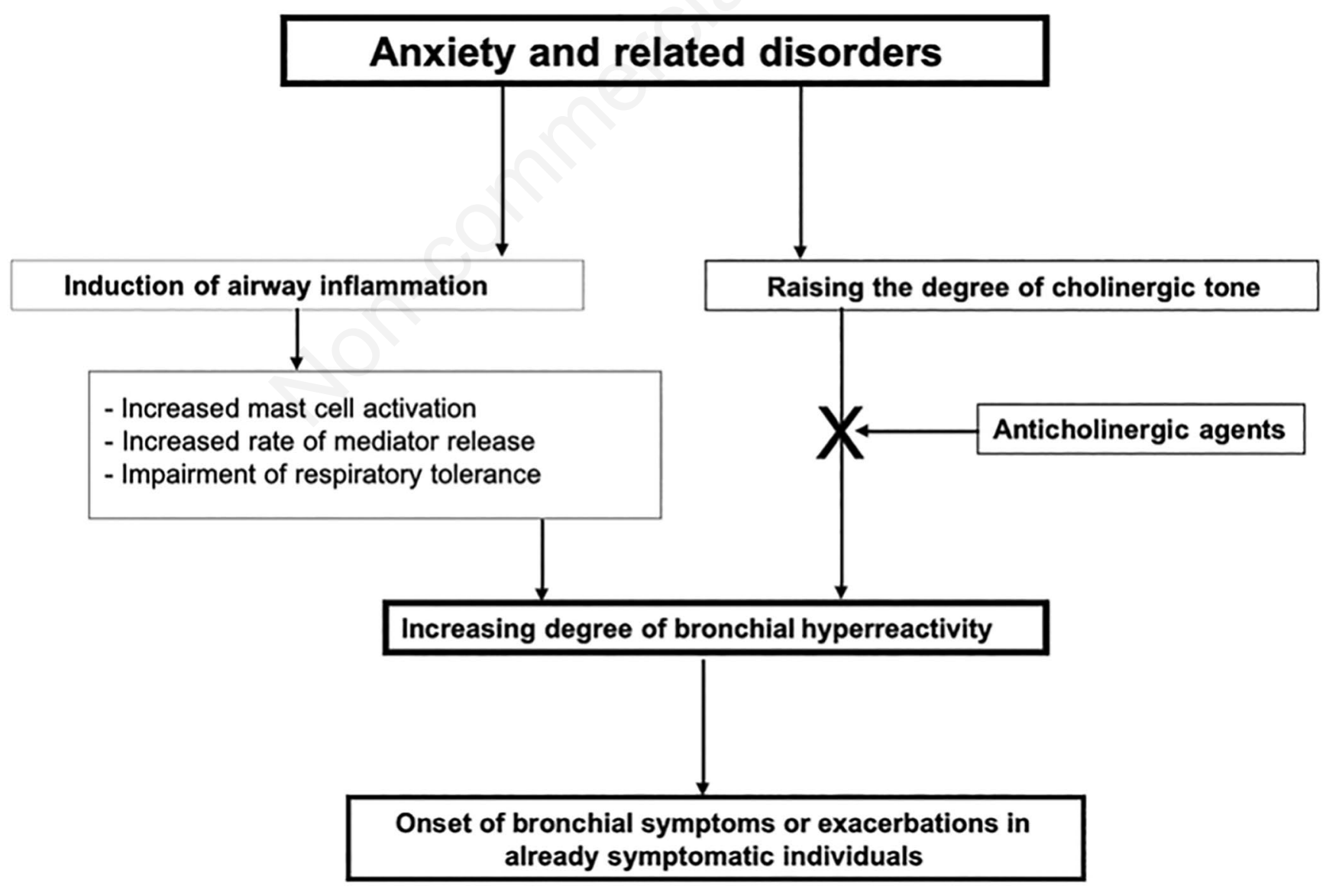

Figure 1. Possible relationship between anxiety/depression and asthma symptoms. 


\section{References}

1. Licari A, Ciprandi R, Marseglia G, Ciprandi G. Anxiety and depression in adolescents with asthma and in their parents: a study in clinical practice. Monaldi Arch Chest Dis 2019; 89:1063.

2. Liccardi G, Baldi G, Berra A, et al. Non-respiratory symptoms

in asthma as possible predictors of exacerbations. J Allergy Clin Immunol Pract 2015;3:798-800.

3. Liccardi G, Salzillo A, Calzetta L, et al. Can bronchial asthma with a highly prevalent airway (and systemic) vagal tone be considered an independent asthma phenotype? Possible role of anticholinergics. Respir Med 2016;117:150-3.

4. Radovanovic D, Santus P, Blasi F, et al. The evidence on tiotropium bromide in asthma: from the rationale to the bedside. Multidiscip Respir Med 2017;12:12. 